Part 3

Sensors 



\section{Polyaniline transmittance spectrum analysis to detection and selectivity of different alcohols and acetic acid}
R. C. Kamikawachi ${ }^{1}$
E. P. M dos Santos ${ }^{1}$
J. F. Kuhne'
E. S. Grizotes ${ }^{1}$
K. K. C. Both'
L. I. Simoes'
J. F. de Deus ${ }^{1}$
P. C. Rodrigues ${ }^{1}$
A. G. Macedo'
K. F. Seidel'

\section{Abstract}

In this work we demonstrate the potential of the polyaniline to the development of a gas sensor exploring the optical properties of a polymer which was verified by UV-Vis spectroscopy measurements. This polymer used as active material, changes its optical properties when exposed to the vapors of different alcohols and acetic acid. The transmittance spectrum of the samples was measured in the range of $350 \mathrm{~nm}$ to $1000 \mathrm{~nm}$ and results show that is it possible to made a selective detection among the isopropanol, ethanol and the acetic acid. Analyses of transmittance measurements as a function of exposure time show an exponential behavior. The fit parameters obtained to the different contaminants are used to prove the response time and sensitivity of the polyaniline film and these can be a indicative to develop a sensor functionalized with this organic material.

Keywords: gas sensor; polyaniline; transmittance spectrum;

\section{Introduction}

Sensors devices is a branch in organic optoelectronic where significant efforts have been done in the last years to extend its application in the areas like safety food, safety in contamination control, clinical diagnostics, etc [1,2]. This sensing can be explored from the point of view of electrical or optical properties of the materials.

1 Federal University of Technology of Paraná - UTFPR, Curitiba, PR-Brazil. 
Conducting polymers presents many vantages to be used in gas sensors if compared to others materials like metal oxides or nanostructures based on carbon. Some advantages are the low cost and the facilities of films preparation, hight sensitivity and short response time, room temperature and non-controlled ambient for operation $[2,3]$.

Among many sensor based on polymers, a big class of that use a polyaniline (PANI) as a active element. In addition to the advantages presented above, normally PANI include a reversible properties providing the possibility of producing a reusable sensor that can be used more than once. Most of the works presented in the literature that use PANI as activity element explores the electrical properties of this polymer like resistance, capacitance, etc. [1,4].

In this work we present the results of changes in the optical properties of the PANI when exposed to organic vapors. We analyzed the transmittance spectrum in the UV-VIS range for isopropanol, ethanol and the acetic acid vapors. The results show that it is possible to obtain a selectivity response for each different substance.

\section{Experimental procedure}

\subsection{Polyaniline synthesis}

The synthesis is based on the chemical oxidative polymerization of aniline in an acidic environment, with $\mathrm{NH} 4 \mathrm{~S} 2 \mathrm{O} 8$ as the oxidant. Instead of using the homogeneous aqueous medium, the polymerization was performed in an immiscible organic/aqueous biphasic system, as described briefly below: aniline was dissolved in toluene, while ammonium peroxydisulfate was dissolved in water with polystyrene sulfonic acid. The two solutions are then carefully transferred to a beaker, generating an interface between the two layers. After $24 \mathrm{~h}$, the entire water phase was filled homogeneously with dark-green polyaniline. The aqueous phase was collected, and the by-products are removed by dialysis against ultrapure water.

\subsection{Sensor development}

The sensors were prepared on a glass microscope slice with dimension of 15 $\mathrm{mm} \times 75 \mathrm{~mm}$ where the PANI solution was deposited by casting without a specifically requirement of deposition as a controlled ambient and/or temperature. After the deposition a thermal treatment was done during 10 minutes at approximately $50^{\circ} \mathrm{C}$ to dry the film. The film is ready to be measured in the sequence. 


\section{Results}

Four samples were prepared to be analyzed where three of them were exposed to the contaminats and the another one never was exposed to any contaminant to give the reference signal. Thus the samples were separately exposed during two minutes to the isopropanol, ethanol and acetic acid vapors. The samples were placed in contact with the contaminant vapor produced by $5 \mathrm{~mL}$ of contaminant placed in a covered beaker of $50 \mathrm{~mL}$ volume.

Immediately in the sequence, we made the first analyses of the transmittance spectrum of the samples in the range of $350 \mathrm{~nm}$ to $1000 \mathrm{~nm}$. The results are showing in the figure 1 where it is possible to observe three different transmittance spectrum. These first analysis shows that the PANI is actuating as active element presenting different values of transmittance when exposed to the contaminant vapors. However, the spectrum analyses shows too a great similarity behavior among the three curves and, mainly, a very small difference between the signal values to the isopropanol and acetic acid.

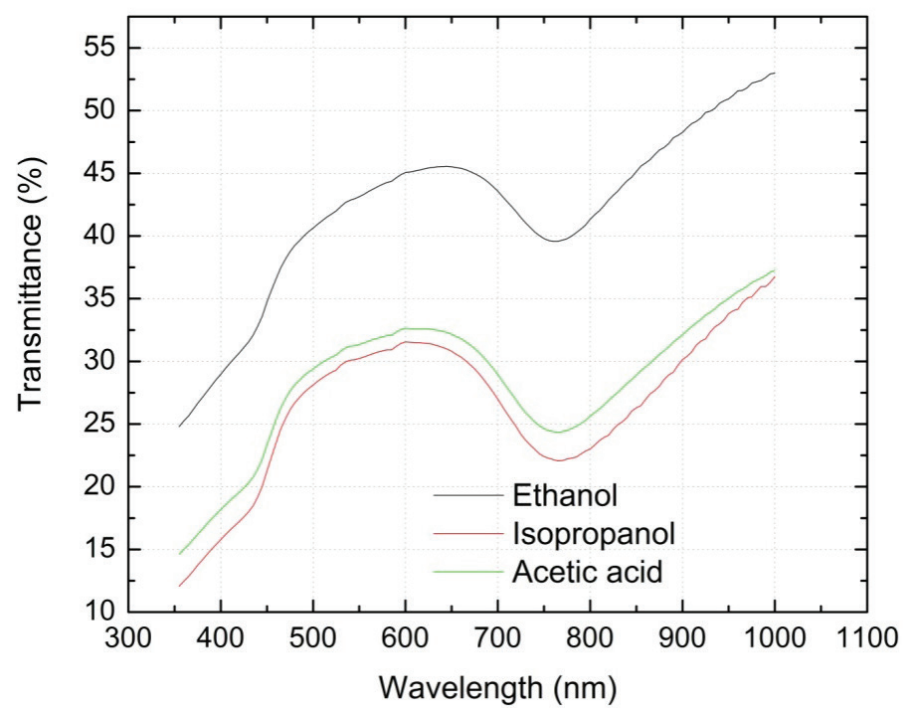

Figure 1 Transmittance versus wave length for three different samples exposed separately to ethanol (black lines), isopropanol (red line) and acetic acid (green line).

The changes in transmittance can be further analyzed by the ratio of the transmittance spectra of the PANI films before and after exposure to vapors. So, the figure 2 shows a specifically selectivity to each contaminant. According to the results, a prudent range to be analyzed where the signal values are shown most differentiated among that is in the range of around $720 \mathrm{~nm}$ to $850 \mathrm{~nm}$. The results 
presented in the figure 2 shows that PANI films provides a selectivity behavior to this detection.

To determine the time response of the sensor, the samples were submitted to the transmittance measures as a function of time. In this measurements the sample stayed in contact with the contaminant vapor during all the measurement time that was $600 \mathrm{~s}$ and the results can be observed in the Figure $3(a, b, c)$. The results present a exponential behavior that was fitted with a exponential decay function (eq. 1).

$$
y=y_{0}+A_{1} e^{\left(-\frac{x}{t_{1}}\right)}
$$

where $\mathrm{y}$ is the transmittance as a function of the time, $y_{0}$ is the minimum transmittance, $y_{0}+A_{1}$ is the maximum transmittance, $A_{1}$ is the transmittance variation amplitude and $t_{1}$ is the decay exponent.

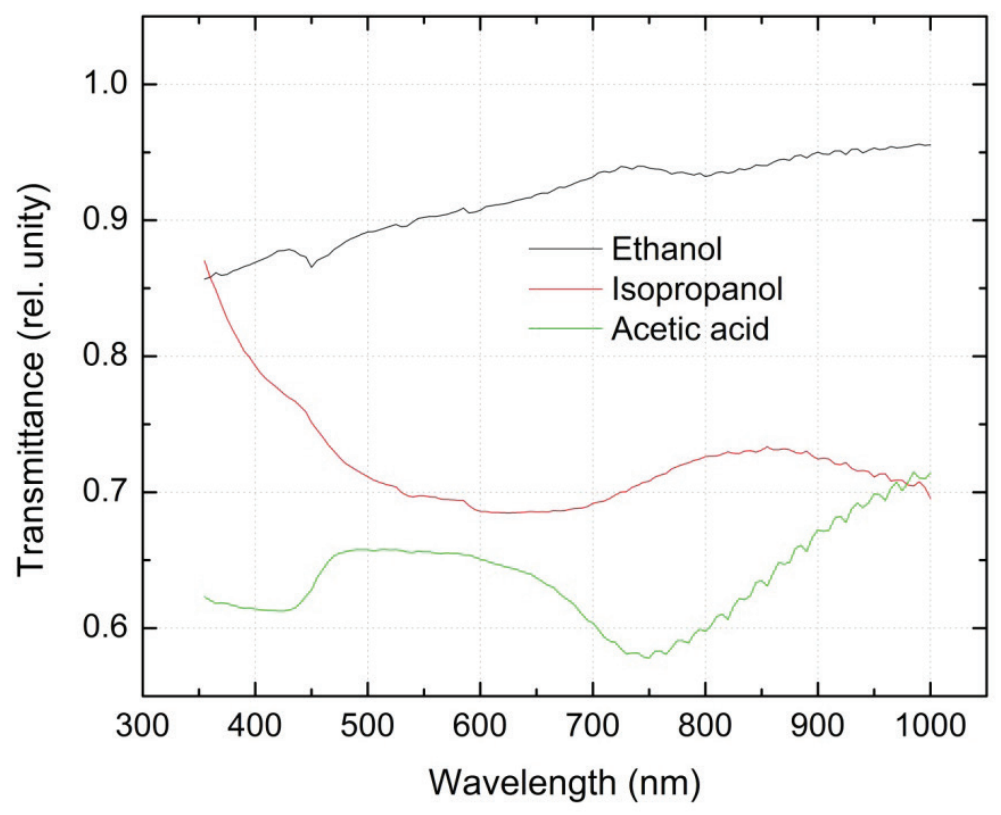

Figure 2 Transmittance spectrum ration of the PANI films before and after exposure to vapors of ethanol (black line), isopropanol (red line) and acetic acid (green line).

The fitting results in the Figure 3 (graphs (a), (b) and (c)) show that the time constant is lower for exposure to isopropanol $(186.8 \pm 1.9) \mathrm{s}$, followed by acetic acid $(206.8 \pm 1.8)$ and ethanol $(238.7 \pm 4.5)$ s decay time. The changes in transmittance was higher to the acetic acid $(0.591 \pm 0.002) \%$ followed by isopropanol $(0.380 \pm 0.001) \%$ and the ethanol $(0.178 \pm 0.001) \%$ with a lower variation 
transmittance. These parameters are indicative of the response time and sensitivity of a sensor functionalized with the a PANI-PSS film.

The samples were exposed to the contaminants and measured for three weeks showing similar results in all the measurements comparisons. That is a great advantage to apply this film to functionalize a sensor due to its signal recovery property.

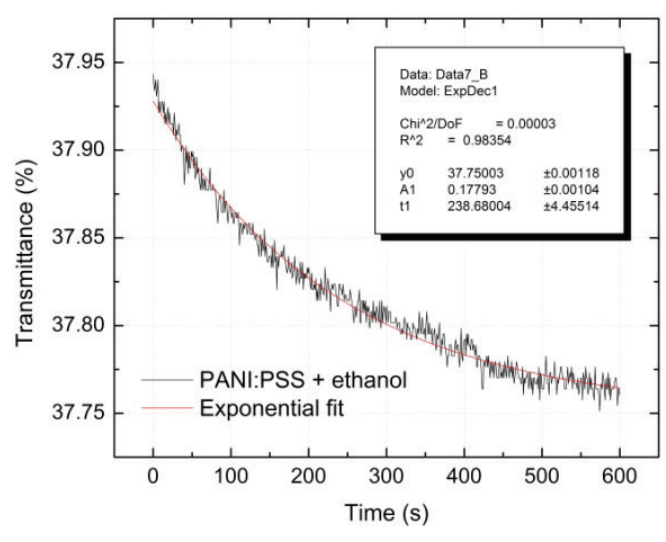

(a)

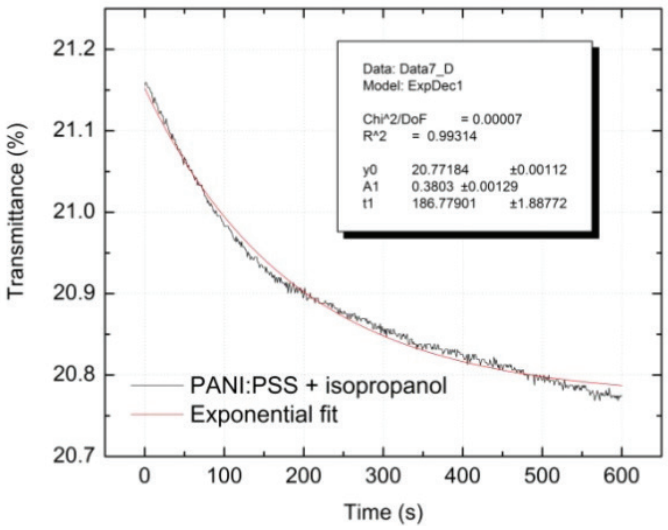

(b)

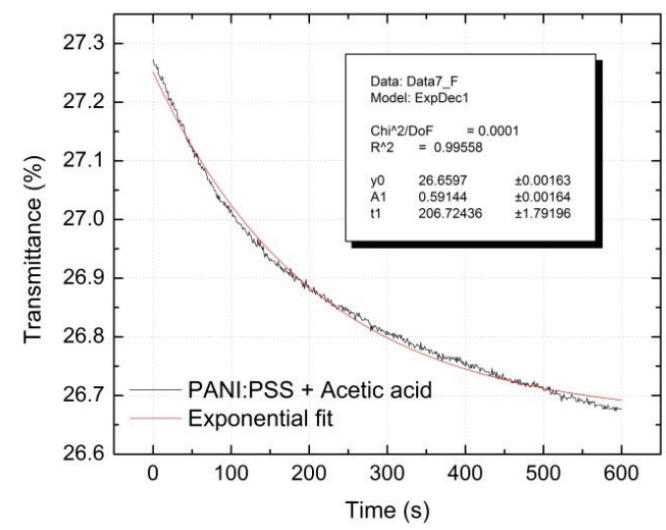

(c)

Figure 3 Transmittance as a function of time of the PANI films during the exposition to the ethanol (a), isopropanol (b) and acetic acid (c).

\section{Conclusion}

We have shown a polymer that presets properties to be used to functionalize a optical sensor. When PANI-PSS film is exposed to isopropanol, ethanol and/ 
or acetic acid its transmittance properties are changed and, therefore, this is a parameter to be explored in a sensor. This parameter can be controlled from the point of view of transmittance spectrum or transmittance as a function of the time where are possible to observe the sensitivity and time response, respectively.

\section{Acknowledgment}

The authors acknowledge FINEP, CAPES, SETI, CNPq and Fundação Araucária for scholarships and funding.

\section{References}

[1] ALVARO DÍAZ AGUILAR, ERICA S. FORZANI, XIULAN LI, NONGJIAN TAO, LARRY A. NAGAHARA, ISLAMSHAH AMLANI AND RAYMOND TSUI. Chemical sensors using peptide-functionalized conducting polymer nanojunction arrays. Appl. Phys. Lett. 87, (2005).

[2] ILARIA FRATODDI, IOLE VENDITTI, CESARE CAMETTI, MARIA VITTORIA RUSSO. Chemiresistive polyaniline-based gas sensors: A mini review. Sensors and Actuators B, 220. (2015).

[3] KELI F. SEIDEL, LUCIELI ROSSI, REGINA M. Q. MELLO, IVO A. HÜMMELGEN. Vertical organic field effect transistor using sulfonated polyaniline/aluminum bilayer as intermediate electrode. J Mater Sci: Mater Electron, 24, (2013).

[4] JUN WANG, SAMUEL CHAN, RICHARD R. CARLSON, YI LUO, GUANGLU GE, RYAN S. RIES, JAMES R. HEATH, HSIAN-RONG TSENG. Electrochemically Fabricated Polyaniline Nanoframework Electrode Junctions that Function as Resistive Sensors. Nano Letters, 4 (9), (2004). 